\title{
Long Josephson junction in a resonant cavity
}

\author{
I. Tornes* and D. Stroud \\ Department of Physics, The Ohio State University, Columbus, Ohio 43210, USA \\ (Received 26 May 2004; revised manuscript received 16 December 2004; published 11 April 2005)
}

\begin{abstract}
We present a model to describe an underdamped long Josephson junction coupled to a single-mode electromagnetic cavity, and carry out numerical calculations using this model in various regimes. The coupling may occur through either the electric or the magnetic field of the cavity mode. When a current is injected into the junction, we find that the time-averaged voltage exhibits self-induced resonant steps (SIRSs) due to coupling between the current in the junction and the electric field of the cavity mode. These steps are similar to those observed and calculated in small Josephson junctions. When a soliton is present in the junction (corresponding to a quantum of magnetic flux parallel to the junction plates), the SIRSs disappear if the electric field in the cavity is spatially uniform. If the cavity mode has a spatially varying electric field, there is a strong coupling between the soliton and the cavity mode. This coupling causes the soliton to become phase locked to the cavity mode, and produces steplike anomalies on the soliton branch of the $I-V$ characteristics. If the coupling is strong enough, the frequency of the cavity mode is greatly redshifted from its uncoupled value. We present simple geometrical arguments which account for this behavior.
\end{abstract}

DOI: 10.1103/PhysRevB.71.144503

PACS number(s): 74.81.Fa, 74.72.- h, 74.25.Nf

\section{INTRODUCTION}

Josephson junction arrays have now been studied for more than 20 years. ${ }^{1}$ They are of interest for many reasons. On a fundamental level, many unusual physical phenomena may occur in them (Kosterlitz-Thouless transitions, and quantum phase transitions from superconductor to insulator). On an applied level, they have been investigated as possible sources of coherent microwave radiation, which would occur if all the junctions in the array were to radiate in phase.

These studies have also been extended to Josephson junctions, and arrays of junctions, coupled to a resonant cavity. When such a system is driven by an applied current, it may exhibit equally spaced self-induced resonant steps (SIRSs) at voltages equal to multiples of $\hbar \Omega / 2 e$, where $\Omega$ is the cavity resonance frequency. ${ }^{2}$ More recently, it was shown that when an array of underdamped junctions is placed in a singlemode cavity, and the array is biased on one of the SIRSs, the array phase locks and radiates energy coherently into the cavity, provided that the number of junctions exceeds a critical threshold. ${ }^{3-6}$ The SIRSs are thought to occur because the cavity acts back on the array like an ac driving field, producing voltage plateaus analogous to Shapiro steps. The physics of the threshold is believed to be that, since each junction is coupled to the same mode, it is effectively coupled to all the other junctions. Thus, there is a long range interaction for which phase locking occurs when the coupling strength, multiplied by the junction number, exceeds a critical value.

Several models have been developed to describe these steps. ${ }^{7-11}$ These models generally succeed in reproducing many of the salient features of the experiments: SIRSs at the expected voltage, and an increase in the energy in the cavity proportional to the square of the number of junctions. The models have now been extended to two-dimensional (2D) junction arrays, ${ }^{12}$ where they show that, on a given step, the $2 \mathrm{D}$ arrays radiate much more energy into the cavity than the one-dimensional (1D) arrays. However, recent work ${ }^{10}$ indi- $^{-}$ cates that, in contrast to experiment, the numerical models always result in the junctions' being phase locked to each other whenever they are biased on a SIRS.

These theories have also been extended into the quantum regime. ${ }^{13-19}$ This regime is relevant when the junctions are very small, so that the noncommutation of the Cooper pair number operator and the phase operator is important. In this regime, the states of the junction (or junctions) and the cavity become entangled, and uniquely quantum phenomena such as Rabi oscillations may be detectable in suitable experiments. Since such junction-cavity systems may be controllable externally, they may be useful as qubits in quantum computation. For this reason, the quantum regime has lately received extensive attention.

Several groups have also investigated the $I-V$ characteristics of a Josephson junction coupled to a phonon mode. This system is formally analogous to the junction-resonant cavity system, in that both involve coupling between a junction and a harmonic oscillator mode. Helm et al. ${ }^{20,21}$ have developed a model describing the coupling between longitudinal phonons and intrinsic Josephson oscillations in cuprate superconductors, which, when sufficiently anisotropic, behave like stacks of underdamped Josephson junctions. ${ }^{22-24}$ These theories have also been extended into the quantum regime. ${ }^{25}$

In all the above calculations, each superconducting island was treated as a small object, with only two degrees of freedom: the phase of the superconducting order parameter, and the number of Cooper pairs on each island. If the island is still large enough for Cooper pair number and phase to commute, then these two degrees of freedom can be treated classically. One then obtains a set of classical coupled secondorder differential equations describing the dynamics of the junction array. For small islands, the phase and number are noncommuting operators, but there are still only two such operators per island.

But one may often be interested in long junctions, and arrays of long junctions. In such systems, the phase difference across the junction typically depends on position along the junction. When such a junction is coupled to a cavity, its 
behavior may therefore differ from that of small junctions.

In this paper, we present a simple model for a long Josephson junction coupled to a single-mode resonant cavity. We present our model in the classical regime, in which number and phase degrees of freedom commute. However, the model should be readily extendible into the quantum regime. We consider both spatially uniform and spatially varying coupling between the junction and the cavity. If the coupling is uniform, we find that, as in the case of small junctions, there are SIRSs in the $I-V$ characteristics provided that there is no magnetic field parallel to the junction plates. But when such a magnetic field is introduced (in the form of a soliton propagating in the junction), the SIRSs are no longer present for uniform coupling. We will give simple arguments why the steps are not to be expected in this case. If the coupling is nonuniform, however, we find that a moving soliton in the long junction does couple to the cavity mode. This coupling perturbs the $I-V$ characteristics of the junction, producing steplike structures in the $I-V$ characteristics, and excitation of the cavity mode. If the coupling is strong enough, this interaction produces a substantial shift in the frequency of the cavity mode. All these perturbations and steps can also be understood by simple physical arguments.

As in the case of short junctions, some previous work has also been done treating the coupling of intrinsic long junctions to phonons. In particular, Preis et al. ${ }^{26}$ have treated this coupling in systems with a magnetic field parallel to the junction plates. They also find resonances and phonon frequency shifts analogous to those found in the present work, although they use a somewhat different mathematical model. Besides this work, several other groups have studied the interaction of solitons in long Josephson junctions with various types of harmonic modes. For example, Kleiner ${ }^{27}$ has investigated the interaction of solitons in stacks of long junctions with the cavity resonances whose frequency and wave number are determined by the stack geometry and by the Josephson plasma frequency. Machida et al. ${ }^{28}$ have numerically studied emission of electromagnetic waves by solitons in stacks of long junctions which are coupled to the Josephson plasma resonances of the stack. Salerno et al. ${ }^{29}$ and Gronbech-Jensen, ${ }^{30}$ have considered the coupling of solitons in long junctions to externally applied microwave fields. Below we compare some of these results with our own.

Since SIRSs may occur in long junctions, they may be useful as sources of sub-THz radiation. Hence, stacks of long junctions may be even more useful as such sources. Since the most anisotropic high- $T_{c}$ cuprate superconductors behave as stacks of long underdamped Josephson junctions, these natural materials, too, may serve as a source of coherent sub- $\mathrm{THz}$ radiation. ${ }^{31}$

The remainder of this paper is arranged as follows. In Sec. II, we derive the equations of motion for treating a long junction coupled to a single-mode resonant cavity. In Sec. III, we present numerical results obtained from these equations of motion, for both no-soliton and soliton initial conditions, and for both uniform and non-uniform junction-cavity coupling. Finally, in Sec. IV, we discuss the numerical results and their experimental implications, and also suggest some possible extensions of this work. A detailed derivation of the Lagrangian for the junction-cavity interaction is presented in the Appendix.

\section{DERIVATION OF EQUATIONS OF MOTION}

\section{A. Lagrangian for a long Josephson junction}

We will consider a long Josephson junction characterized by a gauge-invariant phase difference $\phi(x, y, t)$, where $x$ and $y$ are spatial coordinates and $t$ is the time. $\phi(x, y, t)$ represents the phase difference between the two plates of the junction at points $(x, y)$. We assume that the junction has length $L_{x}$ in the $x$ direction and $L_{y} \gg L_{x}$ in the $y$ direction. For mathematical convenience, we assume periodic boundary conditions in the $x$ direction, so that $\phi\left(x+L_{x}, y, t\right)=\phi(x, y, t)$. We also assume that the phase varies only in the $x$ direction.

In the absence of the cavity, the junction Lagrangian is $L_{\text {junc }}=\int d x \mathcal{L}_{\text {junc }} L_{y}$. The Lagrangian density $\mathcal{L}_{\text {junc }}=K-U$, where $K$ and $U$ are the kinetic and potential energies per unit area. $K=\epsilon d E^{2} /(8 \pi)$ is the energy density stored in the cavity electric field, $d$ is the spacing between the junction plates, and $\epsilon$ is the dielectric constant of the material within the junction.

With the help of the Josephson relation $\phi_{t}=2 \mathrm{eV} / \hbar$ $=2 e d E / \hbar$, where $V$ is the voltage drop across the junction, $e$ is the magnitude of the electronic charge, and the subscript denotes a time derivative, this becomes $K=\epsilon \hbar^{2} \phi_{t}^{2}$ / $8 \pi(2 e)^{2} d$.

The potential energy density $U=U_{J}+U_{B} . \quad U_{J}$ $=-\left(\hbar J_{c} / 2 e\right) \cos \phi$ is the Josephson coupling energy density, $J_{c}$ being the critical current density. The magnetic field energy per unit junction area is

$$
U_{B}=\frac{d B^{2}}{8 \pi},
$$

where $\mathbf{B}=\nabla \times \mathbf{A}$ is the local magnetic induction. The vector potential $\mathbf{A}$ is related to the gauge-invariant phase $\phi$ by $\phi(x, y)=\theta(x, y)-\left(2 \pi / \Phi_{0}\right) \int \mathbf{A}(x, y) d l$, where $\theta$ is the local phase difference across the junction in a particular gauge, $\Phi_{0}=h c /(2 e)$ is the flux quantum, and the second term is the integral of the vector potential across the junction at the point $(x, y)$. We now assume that $\mathbf{B}=B \hat{\mathbf{y}}$, i.e., is parallel to the plates of the junction. If we choose the gauge $\mathbf{A}=-A(x) \hat{\mathbf{z}}$, then $\phi(x, y)=\theta(x, y)+\left(2 \pi / \Phi_{0}\right) A(x) d$ and therefore

$$
B=\frac{\Phi_{0}}{2 \pi d}\left(\phi_{x}-\theta_{x}\right),
$$

where the subscript denotes a partial derivative with respect to $x$.

If the superconductor in the upper plate has a complex order parameter $\Psi_{u}(x, y)=\left|\Psi_{u}\right| \exp \left(i \theta_{u}\right)$, then the corresponding in-plane current density may be written

$$
\mathbf{J}_{u}(x, y)=\frac{e^{*}}{2 m^{*}}\left[\left|\Psi_{u}\right|^{2} \nabla \theta_{u}-\frac{\left(e^{*}\right)^{2}}{m^{*} c} \mathbf{A}\left|\Psi_{u}\right|^{2}\right],
$$

where $e^{*}=2 e, m^{*}$ is the effective mass of a Cooper pair, and the gradient is taken in the xy plane. The coefficient of $\mathbf{A}$ in Eq. (3) can be identified with the London penetration depth $\lambda$ of the material in the upper plate, via the relation $4 \pi\left(e^{*}\right)^{2}\left|\Psi_{u}\right|^{2} /\left(m^{*} c^{2}\right)=1 / \lambda^{2}$. Similar equations hold for $\theta_{\ell}$ on 
the lower plate. Hence, $\nabla \theta \equiv \nabla\left(\theta_{u}-\theta_{\ell}\right)$ satisfies

$$
\nabla \theta=\frac{4 \pi\left(e^{*}\right)^{2} \lambda^{2}}{\hbar c^{2}}\left(\mathbf{J}_{u}-\mathbf{J}_{\ell}\right),
$$

where we have assumed that materials in the upper and lower plates have the same penetration depth. Substituting Eq. (4) into Eqs. (2) and (1), we obtain

$$
U_{B}=\frac{d}{8 \pi}\left[\frac{\Phi_{0} \phi_{x}}{2 \pi d}-\frac{4 \pi \lambda^{2}}{d c}\left(J_{u}-J_{\ell}\right)\right]^{2},
$$

where we have assumed that $\phi$ varies only in the $x$ direction, as is reasonable for $\mathbf{B} \| \hat{\mathbf{y}}$.

Given $\mathcal{L}_{\text {junc }}$, the equations of motion can be obtained from $^{32}$

$$
\frac{\partial}{\partial t} \frac{\partial \mathcal{L}_{\text {junc }}}{\partial \phi_{t}}+\frac{\partial}{\partial x} \frac{\partial \mathcal{L}_{\text {junc }}}{\partial \phi_{x}}-\frac{\partial \mathcal{L}_{\text {junc }}}{\partial \phi}=0
$$

In obtaining these equations and henceforth, we assume $J_{u}$ $-J_{\ell}=0$. The equations then take the form

$$
\phi_{x x}-\frac{1}{\bar{c}^{2}} \phi_{t t}-\frac{1}{\lambda_{J}^{2}} \sin \phi=0,
$$

where $\bar{c}^{2}=c^{2} / \epsilon$ and $1 / \lambda_{J}^{2}=4 \pi e^{*} d J_{c} /\left(\hbar c^{2}\right)$ is the squared Josephson penetration depth. This is the well-known equation for the phase in a long Josephson junction, in the limit of no dissipation. It leads to many solutions, including the sine-Gordon soliton ${ }^{33} \phi(x, t)=4 \tan ^{-1}\left\{\exp \left[\left(x-x_{0}\right) / \lambda_{J}-\beta t\right] /\right.$ $\left.\left(\sqrt{1-\beta^{2}}\right)\right\}$ where $\beta=v / \bar{c}$ is a scaled velocity. This corresponds to an excitation traveling in the positive $x$ direction with speed $v$. This excitation carries a single quantum, $\Phi_{0}$ $=h c / 2 e$ of magnetic flux in the positive $z$ direction. The soliton is relativistically Lorentz contracted and cannot exceed a speed of $\bar{c}$. A similar antisoliton travels with constant speed $v$ in the negative $x$ direction.

\section{B. Cavity Lagrangian}

We assume that the cavity supports one harmonic oscillator mode, described by a "displacement" variable $q_{r}$ and its time derivative $\dot{q}_{r}$. A suitable Lagrangian for this mode is $L_{o s c}=M \dot{q}_{r}^{2} / 2-K q_{r}^{2} / 2$, where $M$ is the "mass" of the oscillator mode and $K$ is the "spring constant." The corresponding Lagrange equation of motion is

$$
\frac{d}{d t}\left(\frac{\partial L_{o s c}}{\partial \dot{q}_{r}}\right)-\frac{\partial L_{o s c}}{\partial q_{r}}=0,
$$

which gives $\ddot{q}_{r}+\Omega^{2} q_{r}=0$, where $\Omega=(K / M)^{1 / 2}$ is the oscillator frequency. Here, we envision the oscillator as an electromagnetic mode of a suitable resonant cavity. In this case, for some types of electromagnetic modes, $q_{r}$ is proportional to the electric field of the mode. The formalism described here would, however, also apply to suitable single-mode mechanical oscillators. With some modifications, it would also apply to electromagnetic modes in which $q_{r}$ represents the magnetic, rather than the electric field.

\section{Cavity-oscillator coupling and bias current}

We assume a capacitive coupling between the junction and the oscillator of the form as is shown in the Appendix, the coupling Lagrangian takes the form

$$
L_{\text {coup }}=-\dot{q}_{r} \int g_{E}(x) \phi_{t}(x) d x L_{y} \equiv \int \mathcal{L}_{\text {coup }} d x L_{y} .
$$

This is a natural extension of coupling assumed in Ref. 9 to a long Josephson junction [see, in particular, Eq. (38) of that paper]. If the cavity electric field is nonuniform, the coupling depends on position along the cavity. In the numerical examples given below, we consider both position-independent and position-dependent coupling.

Besides the capacitive coupling, there could, in principle, also be an inductive coupling between the junction and the cavity mode. In the present work, we do not include this term. However, if the cavity electric field has a nonzero curl, there is a corresponding magnetic field which is already included in Eq. (9). We include this type of electric field in some of our calculations below. A more general derivation of the junction-cavity coupling can be found in the Appendix.

We also need to include a term in the Lagrangian corresponding to the bias current. This takes the form $L_{c u r r}$ $=\left(\hbar J_{z} / 2 e\right) \int d x \phi(x) L_{y}$.

\section{Equations of motion}

The equations of motion are obtained from the analogs of Eqs. (6) and (8), but using the full Lagrangian. We derive the equations of motion assuming a sinusoidal coupling $g_{E}(x)$ $=g_{E} \sin (k x)$, where $g_{E}$ is a constant. The equations of motion for uniform coupling are discussed below.

The total Lagrangian is

$$
L_{\text {tot }}=\int\left(\mathcal{L}_{\text {junc }}+\mathcal{L}_{\text {coup }}\right) d x L_{y}+L_{\text {osc }}+L_{\text {curr }} .
$$

The Lagrange equations of motion take the form

$$
\begin{gathered}
\frac{\partial}{\partial t}\left[\frac{\partial}{\partial \phi_{t}}\left(\mathcal{L}_{\text {junc }}+\mathcal{L}_{\text {coup }}\right)\right]+\frac{\partial}{\partial x}\left[\frac{\partial}{\partial \phi_{x}}\left(\mathcal{L}_{\text {junc }}+\mathcal{L}_{\text {coup }}\right)\right] \\
-\frac{\partial}{\partial \phi}\left(\mathcal{L}_{\text {junc }}+\mathcal{L}_{\text {coup }}\right)=0
\end{gathered}
$$

and

$$
\frac{d}{d t}\left(\frac{\partial L_{t o t}}{\partial \dot{q}_{r}}\right)-\frac{\partial L_{t o t}}{\partial q_{r}}=0 .
$$

Carrying out these operations, we obtain the equations of motion as

$$
\phi_{x x}-\frac{1}{\bar{c}^{2}} \phi_{t t}-\frac{1}{\lambda_{J}^{2}}\left(\sin \phi-\frac{J_{z}}{J_{c}}\right)+g^{\prime} \sin (k x) \ddot{q}_{r}=0,
$$

where

$$
g^{\prime}=\frac{4 \pi d\left(e^{*}\right)^{2}}{\hbar^{2} c^{2}} g_{E}
$$

and 


$$
\ddot{q}_{r}+\Omega^{2} q_{r}=\frac{\hbar^{2} c^{2}}{4 \pi d\left(e^{*}\right)^{2} M} g^{\prime} \int \sin (k x) \phi_{t t} d x L_{y} .
$$

The above equations of motion are derived for a cavity electric field which varies sinusoidally with position. To obtain the equations of motion for a uniform cavity electric field, one simply replaces the term $g^{\prime} \sin (k x)$ in Eqs. (13) and (15) (and subsequent equations) by $g^{\prime}$.

Equations (13) and (15) do not include any damping. We simply incorporate damping by hand, by adding the appropriate terms to these equations. The resulting equations take the form

$$
\phi_{x x}-\frac{1}{\bar{c}^{2}} \phi_{t t}-\frac{\omega_{p}}{Q_{J} \bar{c}^{2}} \phi_{t}-\frac{\omega_{p}^{2}}{\bar{c}^{2}}\left(\sin \phi-\frac{J_{z}}{J_{c}}\right)+g^{\prime} \sin (k x) \ddot{q}_{r}=0
$$

and

$$
\ddot{q}_{r}+\frac{\Omega}{Q_{c}} \dot{q}_{r}+\Omega^{2} q_{r}=\frac{\hbar^{2} c^{2}}{4 \pi d\left(e^{*}\right)^{2} M} g^{\prime} \int \sin (k x) \phi_{t t} d x L_{y} .
$$

Here we have introduced dimensionless junction and cavity quality factors $Q_{J}$ and $Q_{c}$, and a Josephson plasma frequency $\omega_{p}=\bar{c} / \lambda_{J}=\left[4 \pi e^{*} d J_{c} /(\hbar \epsilon)\right]^{1 / 2}$. In terms of the junction parameters, $Q_{J}=\epsilon \omega_{p} / \sigma$, where $\sigma$ is the conductivity of the medium within the junction. The additional terms in Eqs. (16) and (17) ensure that, in the absence of cavity-junction coupling, the equations of motion reduce to the standard results for a long Josephson junction with damping, ${ }^{33}$ and for a damped harmonic oscillator.

\section{E. Reduction to a set of coupled first-order equations}

Equations (16) and (17) are conveniently solved numerically if they are converted into a set of coupled first-order differential equations. Thus, we introduce the momenta canonically conjugate to $q_{r}$ and $\phi$, namely $p_{r}=M \dot{q}_{r}$ $-E_{0} g^{\prime} \int \sin (k x) \phi_{t} d x L_{y}$ and $p_{\phi}=E_{0} \phi_{t} / \bar{c}^{2}-g^{\prime} \sin (k x) E_{0} \dot{q}_{r}$, where $E_{0}=\hbar^{2} c^{2} / 4 \pi d\left(e^{*}\right)^{2}$. Substituting these variables into Eqs. (16) and (17), and rearranging, we obtain the following set of four coupled partial differential equations:

$$
\begin{gathered}
\dot{q}_{r}=\frac{1}{K}\left(\frac{p_{r}}{M}+\frac{\bar{c}^{2} g^{\prime}}{M} \int \sin (k x) p_{\phi} d x L_{y}\right), \\
\dot{p}_{r}=-M \Omega^{2} q_{r}-\frac{M \Omega}{Q_{c}} \dot{q}_{r}, \\
\phi_{t}=\frac{\bar{c}^{2}}{E_{0}} p_{\phi}+g^{\prime} \sin (k x) \bar{c}^{2} \dot{q}_{r}, \\
\left(p_{\phi}\right)_{t}=E_{0} \phi_{x x}-\frac{E_{0} \omega_{p}^{2}}{\bar{c}^{2}}\left(\sin \phi-\frac{J_{z}}{J_{c}}\right)-\frac{\omega_{p} E_{0}}{\bar{c}^{2}} \frac{\phi_{t}}{Q_{J}},
\end{gathered}
$$

where $K=1-\left(E_{0} \bar{c}^{2} g^{\prime 2} L_{x} / M\right) \int \sin ^{2}(k x) d x L_{y}$.

\section{F. Equations of motion in dimensionless form}

It is convenient to rewrite these equations using a dimensionless time $\tau=\omega_{p} t$, length $\xi=x / \lambda_{J}$, and cavity frequency $\widetilde{\Omega}=\Omega / \omega_{p}$. We also introduce the dimensionless variables $\widetilde{p}_{r}$ $=p_{r} /(M \bar{c}), \quad \tilde{p}_{\phi}=\left(M \bar{c}^{2} / E_{0}\right)^{2}\left(p_{\phi} / M \omega_{p}\right) . \quad \tilde{E}_{0}=E_{0} /\left(M \bar{c}^{2}\right), \quad \tilde{q}_{r}$ $=q_{r} / \lambda_{J}$, and finally $\tilde{g}=g^{\prime} L_{y} \bar{c}^{2}$. Finally, we require that $k$ $=2 \pi m / L_{x}$, where $m$ is an integer, consistent with periodic boundary conditions. With these substitutions, and further rearrangement, the equations of motion become

$$
\begin{gathered}
\dot{\tilde{q}}_{r}=\frac{1}{\widetilde{K}}\left(\tilde{p}_{r}+\widetilde{E}_{0}^{2} \tilde{g} \int \tilde{p}_{\phi}\left(\xi_{x}\right) \sin \left(\frac{2 \pi m \xi \lambda_{J}}{L_{x}}\right) d \xi_{x}\right), \\
\dot{\phi}=\widetilde{E}_{0} \tilde{p}_{\phi}+\widetilde{g} \frac{\lambda_{J}}{L_{y}} \dot{\tilde{q}}_{r} \sin \left(\frac{2 \pi m \xi \lambda_{J}}{L_{x}}\right),
\end{gathered}
$$

$$
\begin{gathered}
\tilde{E}_{0} \dot{\tilde{p}}_{\phi}=\phi_{\xi \xi}-\left(\sin \phi-\frac{J_{z}}{J_{c}}\right)-\frac{\dot{\phi}}{Q_{J}}, \\
\dot{\tilde{p}}_{r}=-\frac{\tilde{\Omega}}{Q_{c}} \dot{\tilde{q}}_{r}-\widetilde{\Omega}^{2} \widetilde{q}_{r},
\end{gathered}
$$

where the dot is a derivative with respect to $\tau$ and $\widetilde{K}=1$ $-\left(\widetilde{E}_{0}(\widetilde{g})^{2} / L_{y}\right) \int \sin ^{2}(k x) d x$.

\section{NUMERICAL RESULTS}

\section{A. Algorithm}

We solve Eqs. (19) numerically by discretizing them on a spatial scale of $\Delta$. Thus, $\phi_{\xi \xi} \rightarrow\left[\left(\phi_{j+1}-2 \phi_{j}+\phi_{j-1}\right) / \Delta^{2}\right]$, where $j=1, \ldots, N$, and $N=L_{x} / \Delta \lambda_{J}$ is the number of discrete sections of the long junction. In discretized form, Eqs. (19) become

$$
\begin{gathered}
\dot{\tilde{q}}_{r}=\frac{1}{\tilde{K}}\left[\tilde{p}_{r}+\Delta \widetilde{E}_{0}^{2} \tilde{g} \sum_{i=1}^{N} \tilde{p}_{\phi_{i}} \sin \left(\frac{2 \pi m i}{N}\right)\right], \\
\dot{\phi}_{i}=\widetilde{E}_{0} \tilde{p}_{\phi_{i}}+\widetilde{g} \frac{\lambda_{J}}{L_{y}} \sin \left(\frac{2 \pi m i}{N}\right) \dot{\tilde{q}}_{r}, \\
\widetilde{E}_{0} \dot{\tilde{p}}_{\phi_{i}}=\frac{\phi_{i+1}-2 \phi_{i}+\phi_{i-1}}{\Delta^{2}}-\left(\sin \phi_{i}-\frac{J_{z}}{J_{c}}\right)-\frac{\dot{\phi}_{i}}{Q_{J}}, \\
\dot{\tilde{p}}_{r}=-\frac{\widetilde{\Omega}}{Q_{c}} \dot{\tilde{q}}_{r}-\widetilde{\Omega}^{2} \tilde{q}_{r} .
\end{gathered}
$$

With this discretization, we are basically treating the long junction as $N$ inductively coupled small junctions, each of which has critical current $I_{c}=J_{c}\left(L_{x} L_{y}\right)$.

We have solved Eqs. (20) numerically using a constanttime-step fourth-order Runge-Kutta method with a time step $\Delta \tau=0.001$. We begin the simulation by initializing the variables $\tilde{p}_{r}, \tilde{q}_{r}, \tilde{p}_{\phi_{j}}$ and the parameter $J_{z} / J_{c}$ to zero; we have made various choices for initial values of $\phi_{j}$ as discussed 


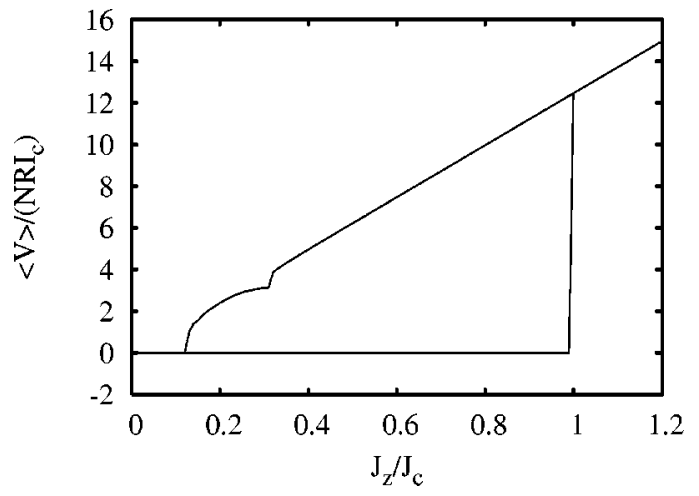

FIG. 1. $I-V$ curve for single long junction, with $Q_{J}=10.0, \Delta$ $=0.05, \widetilde{\Omega}=2.5, \widetilde{E}_{0}^{2} \Delta \widetilde{g}=1.0 \times 10^{-4}, Q_{c}=10$ and all the phases initialized to zero. A SIRS is located at $\langle V\rangle /\left(N R I_{c}\right) \sim \pi$, where $I_{c}$ is the critical current of one of the individual small junctions.

below. For a given $J_{z} / J_{c}$, we integrate the differential equations from $\tau=0$ to $\tau=5 \times 10^{3}$, then evaluate the voltages by averaging over the last $\tau=2 \times 10^{3}$ units of time. The ratio $J_{z} / J_{c}$ is then increased or decreased by 0.01 and the set of equations is solved again. In all cases, we use periodic boundary conditions in the $x$ direction.

We have considered two different initial phase distributions. To model a junction containing no soliton, we choose all the initial phases $\phi_{j}=0$ for $j=1, \ldots, N$. To model a junction containing a single soliton, we assume an initial configuration $\phi_{n}(\tau=0)=2[\pi / 2+\tanh (2 \pi n / N)]$, for $n=1, \ldots, N$. As has been discussed in Ref. 33, for example, these boundary conditions are consistent with the presence of a single soliton, which carries one flux quantum. Our numerical results, as presented below, do show evidence for a soliton with these boundary conditions.

\section{B. Results with no soliton}

In Fig. 1, we show the $I-V$ curve for a single long junction coupled to a cavity, using the no-soliton initial conditions (all phases chosen to equal zero). We choose a position independent junction-cavity coupling with a constant $\widetilde{g} \widetilde{E}_{0}^{2} \Delta=1.0$ $\times 10^{-4}$ and $N=120$. We plot the time-averaged voltages $\langle V\rangle$ in units of $N R I_{c}$, where $I_{c}=J_{c}\left(L_{x} L_{y}\right)$ is the critical current of a single small junction. The $I$ - $V$ characteristics have a step at $\langle V\rangle /\left(N R I_{c}\right)=4 \pi \widetilde{\Omega} / Q_{J}$. This is a SIRS, similar to that seen in individual small junctions for a similar model (see Ref. 9), and occurs at $\langle V\rangle /\left(N R I_{c}\right)=\pi$. This is the voltage expected from the model of Ref. 9, which predicts that the SIRSs will occur when $\langle V\rangle / N R I_{c}$ is an integer multiple of $4 \pi\left(\widetilde{\Omega} / Q_{J}\right)$. The step in Fig. 1 occurs at $4 \pi(2.5 / 10)=\pi$ for $n=1$. On this step, the phases of all the junctions oscillate coherently and are locked onto the cavity mode. To illustrate this coherence, we show in Figs. 2(a) and 2(b) plots of the voltages $V_{i}(\tau) / N R I_{c}$ for small junctions Nos. 59 and 99 over the same time interval. The plots show that the two junctions are indeed oscillating periodically and in phase with one another. In fact, the $V_{i}(\tau) / N R I_{c}$ plots for all the small junctions are identical on this step.
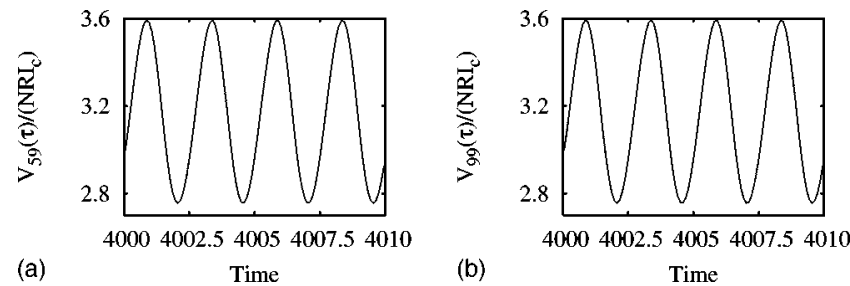

FIG. 2. $V(\tau) /\left(N R I_{c}\right)$ vs dimensionless time $\tau$ for small junctions Nos. (a) 59 and (b) 99 for $4000 \leqslant \tau \leqslant 4010$, plotted for a voltage on the SIRS of Fig. 1.

The phase locking between the junctions and the cavity is illustrated in Fig. 3. This figure shows that the period of oscillation of $\tilde{q}_{r}(\tau)$ is identical to those of $V_{i}(\tau) / N R I_{c}$ shown in Figs. 2; both equal $2 \pi / \widetilde{\Omega}$.

\section{Results with soliton present}

The results shown in Figs. 1-3 are very similar to those obtained in Refs. 9 and 31 for a single small junction coupled to a cavity. However, we see very different results if there is a soliton initially present in the junction. In this case, it is important that the equation of motion for the long junction be discretized on a fine scale. If $N$ is too small, there are spurious steps in the $I-V$ characteristic produced by locking of the soliton to certain linear excitations generated purely by the numerical discretization. These steps, which are numerical artifacts of a too coarse discretization, are well known in real (and discrete) Josephson ladders, and have been discussed extensively. ${ }^{34-36}$ In all our calculations below, $N$ is sufficiently large to avoid these spurious steps.

We discuss first a position-independent coupling between the junction and the cavity. An example of the calculated current-voltage characteristics for this case is shown in Fig. 4. The portion of the $I-V$ characteristic for $J / J_{c} \leqslant 0.6$ corresponds to a soliton which moves freely through the long junction. The soliton behaves like a free, massive, but relativistic, particle, with limiting velocity $\bar{c}$. Its motion is entirely unaffected by coupling to the cavity. The free motion of the soliton can be understood in a simple way by the following argument. If the cavity electric field is uniform, the

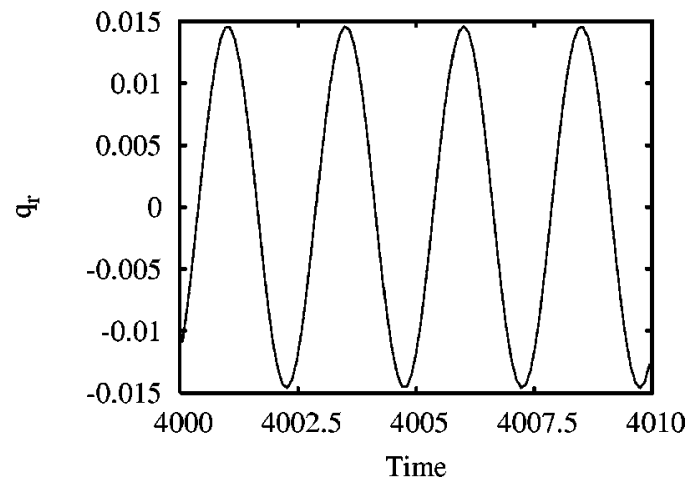

FIG. 3. Cavity variable $q_{r}(\tau)$ for the long junction in Fig. 1, at an applied current on the SIRS, plotted vs time $\tau$ for the time interval $4000 \leqslant \tau \leqslant 4010$. 


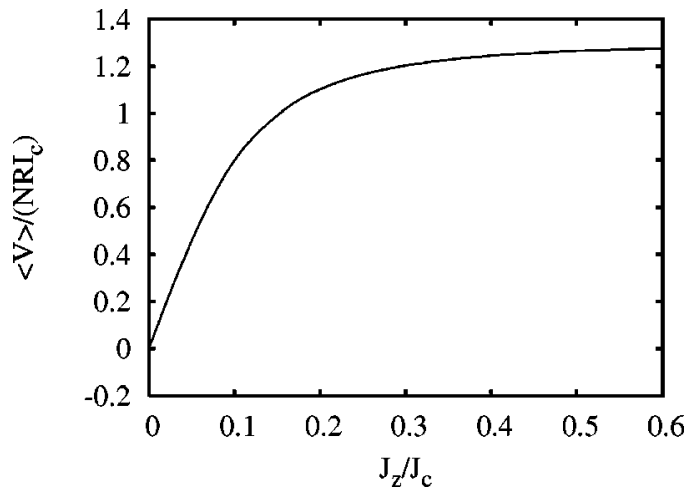

FIG. 4. Current-voltage plot for a single long junction in a resonant cavity, with $Q_{J}=10.0, \Delta=0.05, \widetilde{\Omega}=0.75$, a uniform coupling with $\widetilde{g} \widetilde{E}_{0}^{2} \Delta=1 \times 10^{-4}$, and $Q_{c}=10$. Our initial conditions are such that one soliton is present. This curve is the same for any choice of the strength parameter $\widetilde{g} \widetilde{E}_{0}^{2} \delta$.

coupling between the soliton and the cavity mode is independent of the soliton position. Thus, the cavity electric field exerts no force on the soliton, which therefore should still travel with constant velocity even if there is a strong junction-cavity coupling, consistent with our numerical results. In fact, the $I-V$ characteristics shown in Fig. 4 would be the same if $\widetilde{g}=0$.

Next, we consider a position-dependent junction-cavity coupling. In this case, the $I-V$ characteristics are clearly perturbed by the cavity. Figures 5-7 shows the soliton branch of the full $I-V$ curve for a spatially varying coupling of the form used in Eqs. (16) and (17). By the soliton branch, we mean that part of the $I-V$ curve produced when the current is increased from $J_{z} / J_{c}=0.0$ to approximately 0.6 . We use the same model parameters as for uniform coupling, except that we consider several different coupling strengths, namely $\Delta \tilde{E}_{0}^{2} \tilde{g}=g^{\prime}$, with $g^{\prime}=10^{-4}, 10^{-3}$, and $10^{-2}$ and the dimensionless cavity frequency $\widetilde{\Omega}=0.75$. We also take $m=1$ in Eq. (19). There are now clear steplike structures in the $I-V$ characteristics for all three coupling strengths, at $\langle V\rangle /\left(N R I_{c}\right)$ $\sim 0.95,0.65$, and 0.33 respectively, which were absent in the

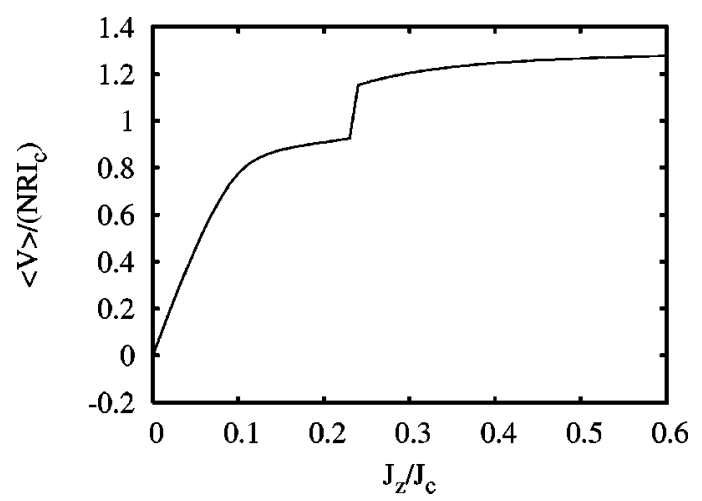

FIG. 5. Current-voltage plot for a single long junction in a cavity, with $Q_{J}=10.0, \Delta=0.05, \widetilde{\Omega}=0.75, N=120, Q_{c}=10$, and coupling $\widetilde{g} \widetilde{E}_{0}^{2} \Delta \sin \left(2 \pi x / L_{x}\right)$, with $\widetilde{g} \widetilde{E}_{0}^{2} \Delta=1.0 \times 10^{-4}$. Our initial conditions are such that one soliton is present.

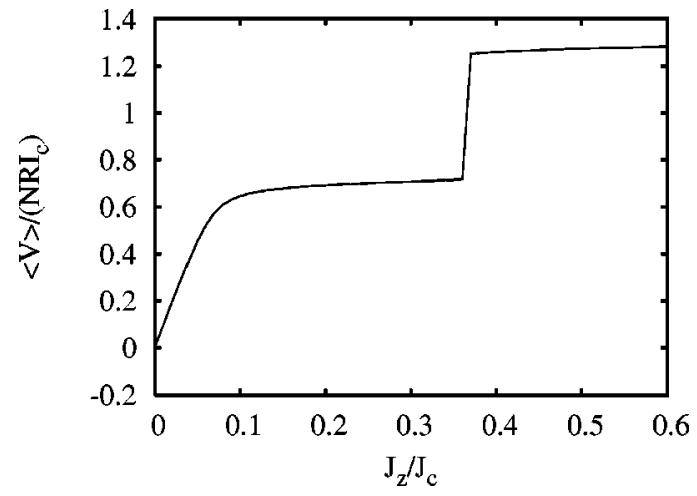

FIG. 6. Same as Fig. 5, except that $\widetilde{g} \widetilde{E}_{0}^{2} \Delta=1.0 \times 10^{-3}$.

case of uniform coupling. These steplike structures correspond in each case to the locking of the soliton to the cavity mode. Specifically, the soliton circulates around the long junction at a frequency of one cycle per cycle of the cavity mode. For the weakest coupling shown, with $g^{\prime}=10^{-4}$, the cavity mode is shifted very little from $\widetilde{\Omega}=0.75$. The voltage step thus occurs approximately at

$$
\frac{\langle V\rangle}{N R I_{c}}=\frac{4 \pi \tilde{\Omega}}{Q_{J}},
$$

or $\langle V\rangle /\left(N R I_{c}\right) \sim 0.95$ for these parameters, corresponding to one cycle of the soliton around the cavity per unperturbed cavity period. For the two stronger couplings shown in Figs. 6 and 7, the soliton is still locked to the cavity, but the coupling is strong enough that the cavity frequency is shifted substantially down from its unperturbed value, to about $2 \widetilde{\Omega} / 3$ and $\widetilde{\Omega} / 3$, respectively. The corresponding timeaveraged voltage on the step is approximately $\langle V\rangle /\left(N R I_{c}\right)$ $=(2 / 3)\left[4 \pi \widetilde{\Omega} / Q_{J}\right] \sim 0.66$, and $(1 / 3)\left[4 \pi \widetilde{\Omega} / Q_{J}\right] \sim 0.33$ for these two couplings.

To see the effects of changing the cavity frequency, we have carried out additional calculations with the same sinusoidal junction-cavity coupling but different cavity frequencies $\widetilde{\Omega}$ and various coupling strengths. The voltage plateaus typically vary approximately linearly with $\widetilde{\Omega}$, as predicted by Eq. (21). An example of this behavior, for the rather large

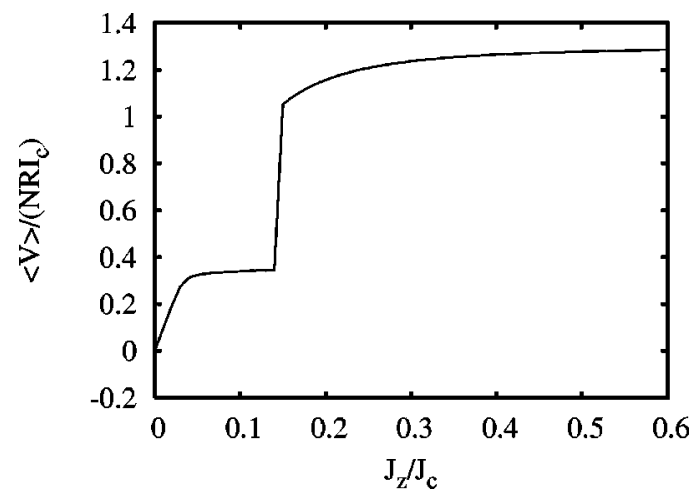

FIG. 7. Same as Fig. 5, except that $\widetilde{g} \widetilde{E}_{0}^{2} \Delta=1.0 \times 10^{-2}$. 


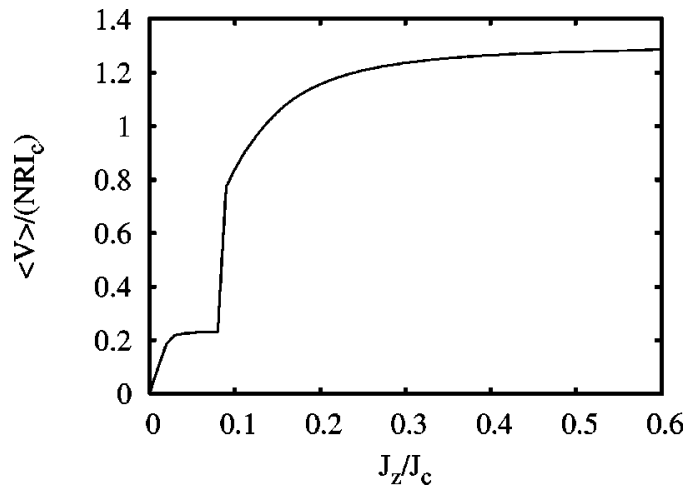

FIG. 8. Same as Fig. 7, except that $\widetilde{\Omega}=0.5$.

coupling constant $g^{\prime}=10^{-2}$, is shown in Fig. 8. $\langle V\rangle /\left(N R I_{c}\right)$ is approximately $2 / 3$ the value of Fig. 7, as suggested by Eq. (21).

Besides the time-averaged voltages, we have also calculated time-dependent voltage differences at various points across the junction for most of the examples shown in Figs. $5-8$, and others. We have also computed the time-dependent cavity variable $q_{r}(\tau)$. In all cases, these calculations provide clear evidence of locking between the junction and the cavity.

Some representative examples of the voltages are shown in Figs. 9-11. In Figs. 9(a), 9(b), 10(a), and 10(b), we show the time-dependent voltages for minijunctions Nos. 60, 120, 30 , and 90 at $J_{z} / J_{c}=0.04$, for the parameters and frequency of Fig. 8. The voltages shown in Figs. 9(a) and 9(b) have the same characteristic shape and period, but are $180 \mathrm{deg}$ out of phase with one another. The same is true for Figs. 10(a) and 10(b). The voltages of the second pair differ in wave form, but not in period, from those of the first pair. Indeed, we have found that all 120 time-dependent voltages on this step have the same period and that the voltages of all minijunction pairs separated by exactly one-half the junction length are identical but $180^{\circ}$ out of phase. The corresponding behavior of $\widetilde{q}_{r}(\tau)$ is shown in Fig. 11. $\tilde{q}_{r}(\tau)$ has the same period as that of all the individual minijunction voltages, showing that the long junction is indeed locked to the cavity mode. Behavior similar to that of Figs. 9 and 10 is also seen in cases with smaller $g^{\prime}$. Because of the weaker coupling, the amplitude of the cavity parameter $\widetilde{q}_{r}$ on these steps (not shown) is much smaller than in Fig. 11. The period of $\widetilde{q}_{r}$ is again the same as that of all the minijunction voltages, and the time-dependent
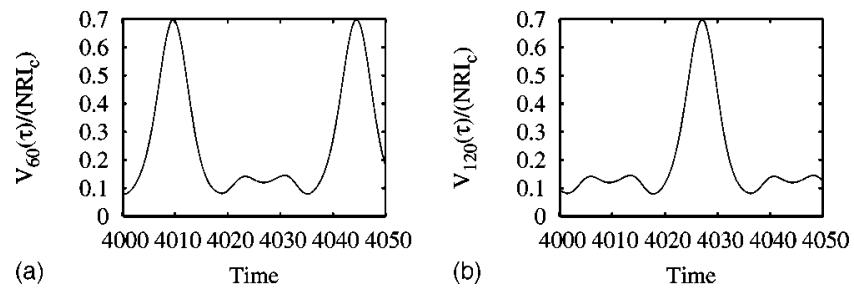

FIG. 9. Time-dependent voltage $V(\tau)$ for small junctions Nos. (a) 60 and (b) 120 , for $4000 \leqslant \tau \leqslant 4050$, plotted vs time $\tau$. Current is such that time-averaged voltage is on the lowest steplike structure of Fig. 8.
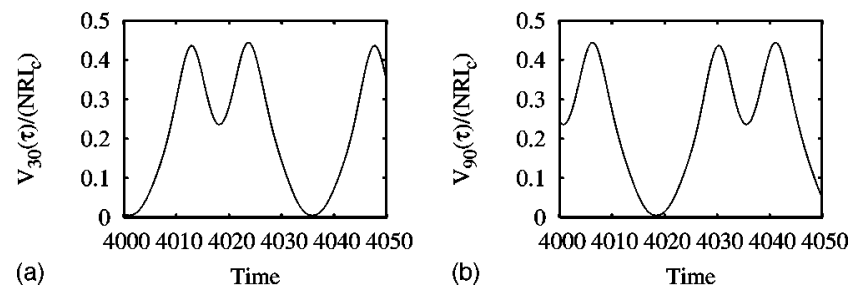

FIG. 10. Same as Figs. 9(a) and 9(b) except that we plot voltages for junctions Nos. (a) 30 and (b) 90 .

voltages are again identical in pairs, but 180 deg out of phase, as in Figs. 9 and 10.

We see from Figs. 5 to 8 that the voltage steps are not completely flat. Despite this slight curvature, we have confirmed numerically that the voltages of all the individual minijunctions have the same period as that of the cavity. Furthermore, the time-averaged voltages across each minijunction are all the same. If we examine the voltages at current values off the step, we find that the cavity and individual junctions no longer have the same periods.

The behavior of the time-dependent voltages at different points along the junction can be understood from the sinusoidal junction-cavity coupling. For every point on the junction, there is a corresponding point separated by $L_{x} / 2$ which experiences an equal and opposite coupling to the cavity. If the coupling has a spatial dependence $\sin \left(2 \pi x / L_{x}\right)$, there are two nodal points along the junction where the coupling is zero, and two points where the coupling has maximum amplitude but is $180^{\circ}$ out of phase. At these two maximum points, the cavity-soliton interaction at any given time is equal in magnitude but opposite in sign. Because of this feature, the time-dependent voltages at these two points should have the same wave form but should be $180^{\circ}$ out of phase, as is seen in Figs. 9(a) and 9(b).

This picture also explains why the time-dependent voltages along the junction are at all points equal in pairs but $180^{\circ}$ out of phase. The different pairs have different voltage wave forms because the coupling amplitude between each minijunction and the cavity varies with the spatial dependence $\sin (k x)$. Despite the different wave forms, we have verified that the time-averaged voltage difference is the same at each point along the junction. This is consistent with the

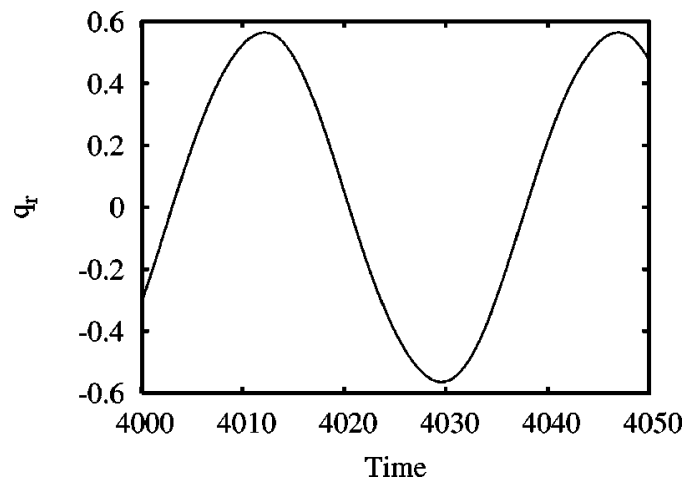

FIG. 11. Cavity variable $q_{r}(\tau)$ for the long junction of Fig. 8, plotted vs $\tau$ for the voltage on the lowest steplike structure for $4000 \leqslant \tau \leqslant 4050$. 


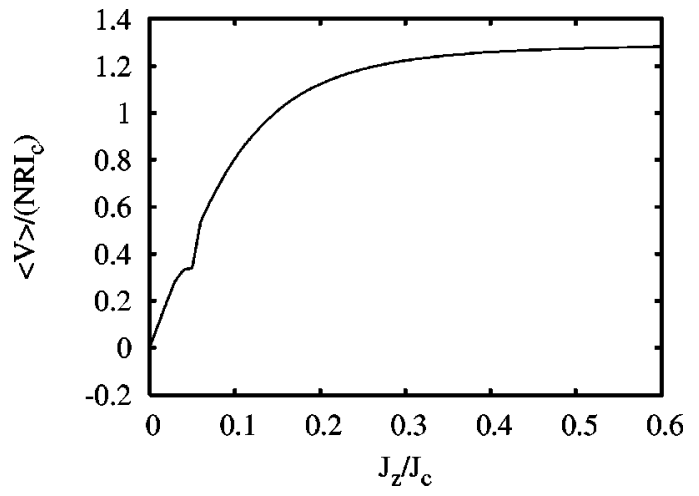

FIG. 12. Current-voltage plot for a single long junction in a cavity, with same parameters as in Fig. 7, except that $\widetilde{\Omega}=1.5$ and the coupling is $\widetilde{g} \widetilde{E}_{0}^{2} \Delta \sin (2 k x)$ with $k=2 \pi / L_{x}$ and $\widetilde{g} \widetilde{E}_{0}^{2} \Delta=1.0$ $\times 10^{-2}$. Our initial conditions are again such that one soliton is present.

picture that this voltage is produced by a soliton which passes each point along the junction with the same average frequency.

Further evidence of a strong soliton-cavity coupling can be seen by comparing Figs. 7 and 12. Figure 7, as noted above, shows the soliton branch of the $I-V$ curve for $\tilde{\Omega}$ $=0.75, \tilde{g} \widetilde{E}_{0}^{2} \Delta=10^{-2}$, and a coupling varying spatially as $\sin \left(2 \pi x / L_{x}\right)$. In the second, we assume the same coupling strength of $\widetilde{g} \widetilde{E}_{0}^{2} \Delta=10^{-2}$ but a frequency $\widetilde{\Omega}=1.5$ and a spatial dependence of $\sin \left(4 \pi x / L_{x}\right)$. In both cases, there are steps at approximately the same value of $\langle V\rangle$, namely $\langle V\rangle /\left(N R I_{c}\right)$ $\sim 0.33 \sim \pi / 10$. These figures show that simultaneously doubling the cavity frequency and halving the coupling periodicity leaves the step height unchanged. We interpret this behavior as showing that, in both cases, the soliton is locked to the cavity mode so that it moves by a distance equal to the wavelength of the mode per mode cycle.

We have also computed $q_{r}(\tau)$ for $J_{z} / J_{c}=0.05$ and the parameters of Fig. 12 (not shown). We find that this amplitude is much smaller than that shown in Fig. 11 for the step of Fig. 7. A simple qualitative explanation for this behavior is the following. The soliton has the same spatial width in both cases, but the junction-cavity coupling varies spatially more rapidly in Fig. 12 than in Fig. 7. Since the junction-cavity coupling thus varies substantially over the width of the soliton in Fig. 12, it has a smaller effect than in Fig. 7. Thus, we expect a much weaker steplike feature in the $I-V$ characteristic of Fig. 12 than in Fig. 7, as is indeed observed numerically.

\section{DISCUSSION}

\section{A. Qualitative argument for soliton steps}

The locking of the moving soliton to the mode of the resonant cavity can be accounted for by a simple analytical argument. The argument starts from the equations of motion (16) and (17). We assume solutions of the form

$$
\phi(x, t)=\phi_{0}+k(x-v t)+\phi_{1} \sin [k(x-v t)],
$$

$$
q_{r}(t)=\operatorname{Re}\left(q_{0} e^{i \omega t}\right) .
$$

This solution is suggested by the fact that, if $g^{\prime}=0$, the solution $\phi(x, t)$ is rigorously of the form $\phi(x, t)=\phi(x-v t)$, where $v$ is the soliton velocity. We now substitute these assumed solutions into Eqs. (16) and (17), carry out the indicated derivatives, and use the standard expansion

$$
\begin{aligned}
\sin \left\{\phi_{0}+k(x-v t)+\phi_{1} \sin [k(x-v t)]\right\} \\
=\sum_{n=-\infty}^{\infty} J_{n}\left(\phi_{1}\right) \sin \left[\phi_{0}+k(x-v t)+n k(x-v t)\right],
\end{aligned}
$$

where $J_{n}\left(\phi_{1}\right)$ is the $n$th order Bessel function. In Eq. (17), we use the expression $\phi_{t t}=-\omega^{2} \phi_{1} \sin [k(x-v t)]$, where $\omega=v k$, and carry out the integral to obtain

$$
\ddot{q}_{r}+\frac{\Omega}{Q_{c}} \dot{q}_{r}+\Omega^{2} q_{r}=-\frac{1}{2} \omega^{2} L_{x} \phi_{1} \cos (\omega t) .
$$

Since this equation is linear, the driven solution for $q_{r}$ is simply

$$
q_{r}(t)=-\frac{1}{2} K g^{\prime} \omega^{2} L_{x} \phi_{1} \operatorname{Re}\left(\frac{e^{i \omega t}}{\Omega^{2}+i \Omega \omega / Q_{c}-\omega^{2}}\right) .
$$

Solution (26) can now be substituted, along with the Bessel function expansion, Eq. (24), back into the other equation of motion, Eq. (16). Next, we assume that the amplitude $\phi_{1}$ is small, and expand the Bessel functions in powers of $\phi_{1}$. Finally, we retain only the lowest Fourier components, namely those involving a constant term, and the functions $\sin [k(x-v t)]$ and $\cos [k(x-v t)]$. A similar approach has been used previously in Refs. 20 and 26 to treat intrinsic Josephson junctions to optical phonons in high-temperature superconductors. Setting the coefficients of each of these terms separately equal to zero in the expanded version of Eq. (16), we finally obtain the following three equations for $\phi_{0}$, $\phi_{1}$, and $\left(J / J_{c}\right)$ :

$$
\begin{gathered}
\frac{1}{\lambda_{J}^{2}} \frac{J}{J_{c}}-\frac{\omega_{p} \omega}{Q_{J} \bar{c}^{2}}+\frac{1}{\lambda_{J}^{2}} \frac{\phi_{1}}{2} \sin \phi_{0}=0 \\
\frac{\omega_{p} \omega}{Q_{J} \bar{c}^{2}} \phi_{1}+\frac{1}{\lambda_{J}^{2}} \sin \phi_{0}+\frac{K^{\prime \prime}\left(\Omega \omega / Q_{c}\right)}{4 D}=0 \\
-k^{2}\left(1-\frac{v^{2}}{\bar{c}^{2}}\right) \phi_{1}+\frac{1}{\lambda_{J}^{2}} \cos \phi_{0}+\frac{K^{\prime \prime}\left(\Omega^{2}-\omega^{2}\right)}{4 D}=0
\end{gathered}
$$

where $\omega=v k, k=2 \pi / L_{x}, K^{\prime \prime}=\left(\hbar^{2} c^{2} g^{\prime}\right)^{2} \omega^{4} L_{x} /\left[16 \pi d\left(e^{*}\right)^{2} M\right]$ and

$$
D=\left(\Omega^{2}-\omega^{2}\right)^{2}+\Omega^{2} \omega^{2} / Q_{C}^{2}
$$

is a resonant denominator. ${ }^{37}$

Equations (28) and (29) are readily solved for $\phi_{0}$ and $\phi_{1}$ in terms of the soliton velocity $v$, and the result substituted into Eq. (27). The resulting Eq. (27) expresses the current $J$ in terms of the soliton velocity $v$, or equivalently, the timeaveraged voltage across the long junction, $\langle V\rangle_{t}$ $=2 \pi \hbar v /\left(2 e L_{x}\right)$. As is suggested by the resonant form of Eqs. (28) and (29), this $I-V$ characteristic has a peak when $\omega$ 
$\sim \Omega$, corresponding to the voltage plateaus observed in our numerical results.

This same approach also shows, through Eq. (26), that there is a peak in the amplitude $q_{r}$ of the cavity mode when the same condition $(\omega=\Omega)$ is satisfied. Once again, this peak in the amplitude is observed in our numerical simulations. For example, Fig. 11 shows the large amplitude of $q_{r}$ at a voltage satisfying the resonance condition; the amplitude of $q_{r}$ at other voltages is much smaller.

\section{B. Comparison to predictions of other models}

It is useful to compare our model and results for the coupled soliton-cavity system to those of other workers. For the case of a uniform coupling, our results are formally analogous to those obtained in Refs. 20 and 21. These workers consider the response of intrinsic Josephson junctions in a high- $T_{c}$ superconductor coupled to an optical phonon mode within the junction. For spatially varying coupling, our numerical results for the locking of a moving fluxon to a cavity mode somewhat resemble those of Refs. 26 and 27, obtained for a coupled fluxon/optical phonon system in an intrinsic Josephson junction, though the equations describing the two systems are not identical. However, there is a significant difference in the physics. The anomalies found in Refs. 26 and 27: in these systems, the quantities which play the role of cavity modes are the optical phonons, which are intrinsic to the junctions themselves. By contrast, our cavity modes are assumed to arise from some cavity extrinsic to the junctions.

\section{Possible realizations of sinusoidal coupling}

Finally, we briefly discuss the type of electromagnetic mode which could produce the sinusoidal coupling we use. The electric field of this mode has a nonzero curl which varies sinusoidally with position, i.e., it has a sinusoidally varying magnetic field. This type of mode should be readily achievable in a real cavity. The voltage steps should be achievable so long as the phase velocity in the cavity is smaller than the limiting soliton velocity $\bar{c}$. Another way to produce this type of coupling would be actually to prepare a long Josephson junction in the shape of a ring, and then to use a cavity mode with a spatially uniform magnetic field. In this case, the junction occupies the ribbonlike region between two circular rings of superconductor; if the planes of the rings are parallel to the $z$ axis, then the magnetic field of the cavity mode should be uniform and parallel to one of the ring diameters. This will produce a flux through the long junction which varies sinusoidally around the ring, as in our model. Measurements using a ring geometry, and a static uniform magnetic field, have recently been reported, in another context, by Wallraff et al., ${ }^{38}$ who also show a schematic picture of this geometry.

\section{SUMMARY}

In this paper, we have described a model for a long underdamped Josephson junction interacting with a singlemode electromagnetic cavity. In our model, we have assumed a capacitive interaction between the junction currents and cavity mode, but we consider both a uniform coupling and one which varies spatially along the junction length. If no soliton (i.e., no fluxon) is present, the junction behaves very much like a small Josephson junction. ${ }^{9}$ In particular, there are SIRSs just as in a small junction, which occur at the voltages expected for a small junction.

If a soliton is present, and the junction-cavity coupling is position independent, then we find that there are no SIRSs. The absence of SIRSs in this case is easily understood: since the soliton-cavity interaction energy is independent of the soliton's position, the cavity exerts no force on the soliton. If, however, the coupling varies sinusoidally with position, we find steplike structure in the $I-V$ characteristics, which arise from the junction-cavity coupling. These structures arise from the locking of the soliton to the cavity mode, so that the soliton travels a distance equal to one wavelength of the coupling interaction during one cycle of the cavity mode, or equivalently, the average soliton velocity equals the phase velocity $\omega / k$ of the cavity mode. We find clear evidence of the locking between the cavity mode and the junction through the time-dependent voltages across the junction. Everywhere along the junction, these time-dependent voltages have the same period of oscillation as the cavity mode. Moreover, the voltage differences of points separated by half a wavelength have exactly the same wave form, but are $180^{\circ}$ out of phase, showing that the soliton travels one wavelength per cycle of the cavity mode.

We have also presented a simple qualitative argument which explains both the positions of the self-induced voltage steps and the occurrence of a peak in the radiated energy on the steps. This argument agrees well with our numerical results in the limit of weak junction-cavity coupling.

If the junction-cavity coupling is strong, this simple argument does not give the position of the anomalies in the $I-V$ characteristics. Instead, the voltage of the steplike structure is shifted substantially down, and the frequency of the cavity mode is strongly redshifted. Nonetheless, the cavity is still locked to the soliton motion.

The voltage on the steps is not absolutely constant, but varies slightly with current. When the current lies on a voltage step, the cavity is strongly excited, with a large timeaveraged squared amplitude $q_{r}^{2}$. If the current does not lie on a step, the cavity and Josephson junction are not locked, and $q_{r}$ is much smaller (at least by an order of magnitude in all our numerical runs). This behavior is once again in agreement with our simple analytical model of the previous section.

The present model could be modified to apply to a stack of long junctions coupled to a single-mode cavity. In the absence of a cavity, it has been known for some time that very anisotropic high- $T_{c}$ cuprate superconductors, such as $\mathrm{BiSr}_{2} \mathrm{Ca}_{2} \mathrm{Cu}_{2} \mathrm{O}_{8+x}$, behave like a stack of underdamped Josephson junctions ${ }^{23}$ Coupling such a stack to a cavity is of great interest because it may provide a means for phase locking these junctions, and hence, providing a coherent source of sub- $\mathrm{THz}$ radiation. The dynamics of a stack of long junctions without a cavity has previously been modeled by Sakai et al..$^{39}$ A stack of long junctions coupled to internal phonon modes within the junctions has been modeled by Preis et al. ${ }^{26}$ The present work suggests a means of modeling 
the coupling of a stack of junctions to the same electromagnetic cavity.

\section{ACKNOWLEDGMENTS}

This work was supported by NSF Grant Nos. DMR0104987 and DMR04-13395. The calculations were carried out using the Ohio Supercomputer facilities. We are very grateful for valuable conversations with Professor B. R. Trees and Dr. E. Almaas.

\section{APPENDIX: ALTERNATE DERIVATION OF A LAGRANGIAN FOR A LONG JUNCTION COUPLED TO A CAVITY}

In this appendix, we present an alternate derivation of the cavity-junction Lagrangian obtained in Sec. II. The derivation here is more general, in that we consider coupling to both the electric and magnetic fields of the cavity.

We write the total Lagrangian as

$$
L=K_{1}-U_{1} \text {. }
$$

The kinetic energy, $K$, is written as

$$
K_{1}=\int d^{3} x \frac{\epsilon(\mathbf{x}) \mathbf{E} \cdot \mathbf{E}}{8 \pi},
$$

where $\epsilon(\mathbf{x})$ is the (possibly position-dependent) dielectric function, and $\mathbf{E}(\mathbf{x})$ is the electric field. We assume that the electric field is the sum of two parts: that due to the junction, which we denote $\mathbf{E}_{\text {junc }}$, and the part due to cavity mode, which we write as $\mathbf{E}_{c a v}$. The junction field takes the form

$$
\mathbf{E}_{\text {junc }}=\frac{\hbar}{2 e d} \phi_{t} \hat{\mathbf{z}} \text {. }
$$

Then $K_{1}$ takes the form

$$
\begin{aligned}
K_{1}= & L_{y} \int d x \frac{\epsilon \hbar^{2} \phi_{t}^{2}}{8 \pi(2 e)^{2} d}+\frac{1}{8 \pi} \int d^{3} x \epsilon(\mathbf{x})\left(\mathbf{E}_{c a v} \cdot \mathbf{E}_{c a v}\right) \\
& +\frac{1}{4 \pi} \int d^{3} x \epsilon(\mathbf{x}) \mathbf{E}_{c a v}(\mathbf{x}) \cdot \frac{\hbar}{2 e d} \phi_{t} \hat{\mathbf{z}} .
\end{aligned}
$$

Here the volume integral is taken over the cavity, and we are assuming a geometry such that the junction is contained within the cavity.

The potential energy $U_{1}$ is the sum of two terms. The first is the Josephson energy

$$
U_{1, J}=-L_{y} \int d x \frac{\hbar J_{c}}{2 e} \cos \phi .
$$

The other part is the energy of the magnetic field. This may be written

$$
U_{1, \text { mag }}=\int d^{3} x \frac{\mathbf{B} \cdot \mathbf{B}}{8 \pi} .
$$

Once again, the magnetic field may be written

$$
\mathbf{B}=\mathbf{B}_{\text {junc }}+\mathbf{B}_{\text {cav }},
$$

where $\mathbf{B}_{\text {junc }}$ and $\mathbf{B}_{\text {cav }}$ are the fields due to the junction and cavity. As in the text, we again assume that $J_{u}=J_{\ell}$, so that

$$
\mathbf{B}_{\text {junc }}=\frac{\Phi_{0} \phi_{x}}{2 \pi d} \hat{\mathbf{y}} .
$$

Then the magnetic field energy takes the form

$$
\begin{aligned}
U_{1, \text { mag }}= & L_{y} \int d x \frac{\Phi_{0}^{2} \phi_{x}^{2}}{32 \pi^{3} d}+\frac{1}{8 \pi} \int d^{3} x \mathbf{B}_{c a v} \cdot \mathbf{B}_{c a v} \\
& +L_{y} \int d x \frac{1}{4 \pi} \frac{\Phi_{0} \phi_{x}}{2 \pi} \hat{y} \cdot \mathbf{B}_{c a v}(\mathbf{x}) .
\end{aligned}
$$

To make further progress, we introduce operators describing the fields $\mathbf{E}_{c a v}$ and $\mathbf{B}_{c a v}$. Both may be expressed in terms of the operator for the cavity vector potential

$$
\mathbf{A}_{c a v}(\mathbf{x}, t)=\left(\frac{h c^{2}}{\Omega}\right)^{1 / 2}\left[a(t)+a^{\dagger}(t)\right] \mathbf{E}(\mathbf{x})
$$

Here $a$ and $a^{\dagger}$ are the annihilation and creation operators for the cavity mode, which satisfy the usual Bose commutation relations

$$
\left[a, a^{\dagger}\right]=1 \text {. }
$$

$\mathbf{E}(\mathbf{x})$ is proportional to the position-dependent electric field of the cavity mode; its normalization is given below. $\Omega$ is the frequency of the cavity mode. In terms of this operator, the electric field operator $\mathbf{E}_{c a v}(\mathbf{x}, t)$ is given by

$$
\mathbf{E}_{c a v}(\mathbf{x}, t)=-\frac{1}{c} \frac{\partial \mathbf{A}_{c a v}}{\partial t}=-i(h \Omega)^{1 / 2}\left(a-a^{\dagger}\right) \mathbf{E}(\mathbf{x})
$$

and the magnetic field operator $\mathbf{B}_{\text {cav }}(\mathbf{x}, t)$ is

$$
\mathbf{B}_{c a v}(\mathbf{x}, t)=\nabla \times \mathbf{A}_{c a v}=\left(\frac{h c^{2}}{\Omega}\right)^{1 / 2}\left[a+a^{\dagger}\right] \nabla \times \mathbf{E}(\mathbf{x})
$$

The operator describing the total energy in the cavity is

$$
W_{c a v}=\int d^{3} x \frac{\mathbf{E}_{c a v} \cdot \mathbf{E}_{c a v}+\mathbf{B}_{c a v} \cdot \mathbf{B}_{c a v}}{8 \pi} .
$$

We calculate the ensemble average of this operator, using the results $\left\langle a^{\dagger} a\right\rangle=n ;\left\langle a a^{\dagger}\right\rangle=n+1 ;\langle a a\rangle=\left\langle a^{\dagger} a^{\dagger}\right\rangle=0$, with the result

$$
\left\langle W_{c a v}\right\rangle=\frac{1}{2}\left(n+\frac{1}{2}\right) \hbar \Omega \int\left[|\mathbf{E}|^{2}+\frac{c^{2}}{\Omega^{2}}|\nabla \times \mathbf{E}|^{2}\right] d^{3} x .
$$

In order for this energy to equal $\hbar \Omega\left(n+\frac{1}{2}\right)$, we require that the function $\mathbf{E}(\mathbf{x})$ be normalized so that

$$
\int\left[|\mathbf{E}|^{2}+\frac{c^{2}}{\Omega^{2}}|\nabla \times \mathbf{E}|^{2}\right] d^{3} x=2
$$

Also, we assume that the cavity energy is equally distributed between the electric and magnetic fields. This implies that 


$$
\int|\mathbf{E}|^{2} d^{3} x=\frac{c^{2}}{\Omega^{2}} \int|\nabla \times \mathbf{E}|^{2} d^{3} x=1 .
$$

Having obtained the operator forms for the cavity electric and magnetic fields, we are now in a position to derive the electric and magnetic parts of the junction-cavity coupling. After some algebra, the electric field part of the coupling Lagrangian may be written

$$
L_{\text {coup }, E}=\frac{1}{4 \pi} L_{y} \int d x \frac{\hbar}{2 e} \phi_{t}\left[-i(h \Omega)^{1 / 2}\left(a-a^{\dagger}\right)\right] E_{z}(\mathbf{x}) .
$$

Similarly, the magnetic field part of the coupling Lagrangian may be written

$$
L_{\text {coup }, B}=\frac{1}{4 \pi} L_{y} \frac{\Phi_{0}}{2 \pi}\left(h c^{2} / \Omega\right)^{1 / 2} \int d x\left(a+a^{\dagger}\right) \phi_{x}[\nabla \times \mathbf{E}(\mathbf{x})]_{y} .
$$

To make contact with the notation in the main part of this paper, we introduce position and momentum operators for the cavity mode, by

$$
p_{r}=\left(\frac{M \hbar \Omega}{2}\right)^{1 / 2} i\left(a^{\dagger}-a\right)
$$

and

$$
q_{r}=\left(\frac{\hbar}{2 M \Omega}\right)^{1 / 2}\left(a+a^{\dagger}\right) .
$$

These operators have the standard canonical commutation relations

$$
\left[p_{r}, q_{r}\right]=-i \hbar .
$$

In terms of these operators, we may write the Hamiltonian for the cavity alone as

$$
H_{c a v}=\frac{1}{8 \pi} \int\left(\mathbf{E}_{c a v} \cdot \mathbf{E}_{c a v}+\mathbf{B}_{c a v} \cdot \mathbf{B}_{c a v}\right)=\frac{p_{r}^{2}}{2 M}+\frac{K q_{r}^{2}}{2},
$$

where we define the "spring constant"

$$
K=M \Omega^{2} .
$$

From $H_{c a v}$ we may infer the corresponding cavity Lagrangian using $q_{r}=p_{r} / M$, with the result

$$
L_{c a v}=\frac{M \dot{q}_{r}^{2}}{2}-\frac{1}{2} K q_{r}^{2} .
$$

It is readily verified that classical Hamiltonian equations of motion resulting from the Hamiltonian (A21) are equivalent to the classical Lagrange equation of motion obtained from the Lagrangian (A23); both lead to

$$
\ddot{q}_{r}-\Omega^{2} q_{r}=0 .
$$

Finally, we can express the coupling Lagrangians in terms of the variables introduced above. First $L_{\text {coup , }}$ takes the form

$$
L_{\text {coup }, E}=L_{y} \int d x \mathcal{L}_{\text {coup }, E}(x),
$$

where

$$
\mathcal{L}_{\text {coup }, E}(x)=-g(x) \dot{q}_{r} \phi_{t},
$$

and

$$
g(x)=-\epsilon \sqrt{\frac{M}{4 \pi}} \frac{\hbar}{2 e} \mathbf{E}(x) \cdot \hat{\mathbf{z}} .
$$

Similarly, $L_{\text {coup }, B}$ takes the form

$$
L_{\text {coup }, B}=-L_{y} \int d x \mathcal{L}_{\text {coup }, B}(x),
$$

where

$$
\mathcal{L}_{\text {coup }, B}(x)=-g_{B}(x) q_{r} \phi_{x},
$$

and

$$
g_{B}(x)=\sqrt{\frac{M c^{2}}{16 \pi^{3}}} \Phi_{0} \nabla \times \mathbf{E} \cdot \hat{\mathbf{y}} .
$$

There is one additional term in the Lagrangian which also represents a coupling between the cavity and the junction. This is the term

$$
L_{d}=L_{y} \int d x \frac{d}{8 \pi}(\epsilon-1) \mathbf{E}_{c a v} \cdot \mathbf{E}_{c a v} .
$$

But this term does not couple the cavity variables to those of the junction. Instead, its only effect will be to produce a slight shift in the cavity resonance frequency. We have therefore not included this term in our calculations described in the body of the paper.
*Email address: itornes@mps.ohio-state.edu

†Email address: stroud@mps.ohio-state.edu

${ }^{1}$ For a recent review, see, e.g., C. J. Lobb and R. S. Newrock, in Solid State Physics, edited by H. Ehrenreich and F. Spaepen (2000), Vol. 54, p. 263

${ }^{2}$ A. Larsen, H. D. Jensen, and J. Mygind, Phys. Rev. B 43, 10179 (1991).
${ }^{3}$ P. Barbara, A. B. Cawthorne, S. V. Shitov, and C. J. Lobb, Phys. Rev. Lett. 82, 1963 (1999).

${ }^{4}$ B. Vasilić, P. Barbara, S. V. Shitov, and C. J. Lobb, IEEE Trans. Appl. Supercond. 11, 1188 (2001).

${ }^{5}$ B. Vasilić, P. Barbara, S. V. Shitov, and C. J. Lobb, Phys. Rev. B 65, 180503(R) (2002).

${ }^{6}$ B. Vasilić, E. Ott, T. Antonsen, P. Barbara, and C. J. Lobb, Phys. 
Rev. B 68, 024521 (2003).

${ }^{7}$ G. Filatrella, N. F. Pedersen, and K. Wiesenfeld, Phys. Rev. E 61, 2513 (2000).

${ }^{8}$ G. Filatrella, N. F. Pedersen, and K. Wiesenfeld, IEEE Trans. Appl. Supercond. 11, 1184 (2001).

${ }^{9}$ E. Almaas and D. Stroud, Phys. Rev. B 65, 134502 (2002).

${ }^{10}$ G. Filatrella, N. Falsic Pedersen, C. J. Lobb, and P. Barbara, Eur. Phys. J. B 34, 3 (2003).

${ }^{11}$ R. Bonifacio, F. Casagrande, and M. Milani, Lett. Nuovo Cimento Soc. Ital. Fis. 34, 520 (1982).

${ }^{12}$ E. Almaas and D. Stroud, Phys. Rev. B 67, 064511 (2003).

${ }^{13}$ A. Shnirman, G. Schön, and Z. Hermon, Phys. Rev. Lett. 79, 2371 (1997).

${ }^{14}$ O. Buisson and F. W. J. Hekking, cond-mat/0008275 (unpublished).

${ }^{15}$ A. Blais, R.-S. Huang, A. Wallraff, S. M. Girvin, and R. J. Schoelkopf, Phys. Rev. A 69, 062320 (2004).

${ }^{16}$ M. J. Everitt, P. Stiffell, T. D. Clark, A. Vourdas, J. F. Ralph, H. Prance, and R. J. Prance, Phys. Rev. B 63, 144530 (2001).

${ }^{17}$ W. A. Al-Saidi and D. Stroud, Phys. Rev. B 65, 014512 (2002); 65, 224512 (2002).

${ }^{18}$ A. Blais, A. Maassen van den Brink, and A. M. Zagoskin, Phys. Rev. Lett. 90, 127901 (2003).

${ }^{19}$ J. Q. You and F. Nori, Phys. Rev. B 68, 064509 (2003).

${ }^{20}$ C. Helm, C. Preis, F. Forsthofer, J. Keller, K. Schlenga, R. Kleiner, and P. Müller, Phys. Rev. Lett. 79, 737 (1997).

${ }^{21}$ C. Helm, C. Preis, C. Walter, and J. Keller, Phys. Rev. B 62, 6002 (2000).

${ }^{22}$ R. Kleiner, P. Müller, H. Kohlstedt, N. F. Pedersen, and S. Sakai, Phys. Rev. B 50, 3942 (1994).

${ }^{23}$ R. Kleiner, F. Steinmeyer, G. Kunkel, and P. Müller, Phys. Rev. Lett. 68, 2394 (1992).
${ }^{24}$ R. Kleiner and P. Müller, Phys. Rev. B 49, 1327 (1994).

${ }^{25}$ See, e.g., E. K. Irish and K. Schwab, Phys. Rev. B 68, 155311 (2003); X. B. Zou and W. Mathis, Phys. Lett. A 324, 484 (2004); M. Blencowe, Phys. Rep. 395, 159 (2004); I. Martin, A. Shnirman, L. Tian, and P. Zoller, Phys. Rev. B 69, 125339 (2004).

${ }^{26}$ C. Preis, C. Helm, K. Schmalzl, J. Keller, R. Kleiner, and P. Müller, Physica C 362, 51 (2001).

${ }^{27}$ R. Kleiner, Phys. Rev. B 50, 6919 (1994).

${ }^{28}$ M. Machida, T. Koyama, and M. Tachiki, Physica C 362, 16 (2001).

${ }^{29}$ M. Salerno, M. R. Samuelsen, G. Filatrella, S. Pagano, and R. D. Parmentier, Phys. Rev. B 41, 6641 (1990).

${ }^{30}$ N. Gronbech-Jensen, Phys. Rev. B 47, 5504 (1993).

${ }^{31}$ I. Tornes and D. Stroud, Phys. Rev. B 68, 052512 (2003).

${ }^{32}$ See, e.g., A. L. Fetter and J. D. Walecka, Theoretical Mechanics of Particles and Continua (McGraw-Hill, New York, 1980), Eq. (25.59).

${ }^{33}$ A. V. Ustinov, M. Cirillo, and B. A. Malomed, Phys. Rev. B 47, 8357 (1993).

${ }^{34}$ H. S. J. van der Zant, T. P. Orlando, S. Watanabe, and S. H. Strogatz, Phys. Rev. Lett. 74, 174 (1995).

${ }^{35}$ S. Watanabe, S. H. Strogatz, H. S. J. van der Zant, and T. P. Orlando, Phys. Rev. Lett. 74, 379 (1995).

${ }^{36}$ For a review, see, e.g., A. V. Ustinov, Physica D 123, 315 (1998).

${ }^{37}$ In obtaining these equations, we have written terms of the form $\sin (k x) \cos (\omega t)=\frac{1}{2}[\sin (k x-\omega t)+\sin (k x+\omega t)]$ and $\sin (k x) \sin (\omega t)$ $=\frac{1}{2}[\cos (k x-\omega t)-\cos (k x+\omega t)]$ and neglected the "counterrotating" terms $\cos (k x+\omega t)$ and $\sin (k x+\omega t)$.

${ }^{38}$ A. Wallraff, A. Lukashenko, J. Lisenfeld, A. Kemp, M. V. Fistul, Y. Koval, and A. V. Ustinov, Nature (London) 425, 155 (2003).

${ }^{39}$ S. Sakai, P. Bodin, and N. F. Pedersen, J. Appl. Phys. 73, 2411 (1993). 